\title{
Battlefield Advanced Training Life Support
}

Appendix 1
BATLS/BARTS Glossary of
Terms $^{1,2}$

a- Prefix meaning without or absent.

ab- Prefix meaning away from (for example, abduct).

abdomen The portion of the body between the thorax and the pelvis containing the majority of the organs of digestion as well as the liver and spleen.

abduct To move away from the midline of the body.

ABO blood groups One of a number of classifications of blood types based on the presence of the protein groups A or B on the red corpuscles. Groups are divided into $\mathrm{A}$ (the presence of group A proteins), B (the presence of group $\mathrm{B}$ proteins), $\mathrm{AB}$ (both $\mathrm{A}$ and $\mathrm{B}$ present) or $\mathrm{O}$ (no antigens present). Normally, blood requires cross-matching into at least a similar blood group between casualty and donor before transfusion, though in a desperate emergency blood from group $\mathrm{O}$ may be given to any casualty.

acidosis The condition of an excess concentration of hydrogen ions in the body. (This is the opposite of alkalosis.) This may be as a result of either a metabolic problem or a respiratory problem. Treatment relies on correcting the cause of the imbalance.

acute hypoxia Sudden fall in oxygen concentration within the blood stream. It may be caused by airway obstruction, lung failure or cardiac failure. Signs may be subtle such as confusion or more obvious such as acute loss of consciousness.

ad- Prefix meaning towards (for example, adduct).

airway The passage allowing free movement of air into and out of the lungs. The term is also used to describe clinical devices for maintenance of a patient airway; these may range from simple devices such as Guedel airways to endotracheal tubes.

alkalosis The condition of a decrease in the body's concentration of hydrogen ions or an increase in bicarbonate ions. Like acidosis, this may be caused by respiratory (hyperventilation) or metablic causes (the ingestion of excess bicarbonate or loss of excess gastric acid following vomiting). allergy Abnormal sensitivity to normally harmless antigens. In the severest form this may result in an anaphylactic reaction. alveolus (Latin, a small hollow) the small sac-like terminations of lung tissue at which level gas exchange takes place.

amputation The loss of a digit, limb or appendage, either deliberately (surgically) or as a result of trauma.

anaemia An abnormally low haemoglobin concentration in the blood.

anaesthesia (Greek, absence of feeling) A loss or normal sensation, particularly pain. This may be as a result of disease or induced deliberately. Anaesthesia may be local (by injecting local anaesthetic drugs around an area), regional (by blocking a nerve or nerves supplying a region) or general, which normally involves reducing a patient to a state of unconsciousness.

analgesic A pain-relieving drug.

anaphylactic reaction An extreme reaction to an allergen characterised by release of histamine from cells of the immune system and resulting in generalised itching, angioedema, collapse, tachycardia, bronchospasm and (in the worst cases) death.

Angle of Louis The prominence of the sternum opposite the second intercostal cartilage.

antecubital The anatomical area at the front of the elbow (for example, antecubital fossa).

anterior In front of or at the forward limits of the body.

antibiotic A drug with antibacterial actions. antigen $A$ substance, usually a protein, which the body regards as foreign and against which it produces an antibody in an attempt to render the antigen harmless.

aorta The main arterial supply to the body. It commences at the outflow of the left ventricle as the ascending aorta and arches within the chest cavity (aortic arch) to give off the main vascular supply to the head. It then descends through the thorax (thoracic aorta) and into the abdomen (abdominal aorta) to supply the intestines before splitting into two terminal branches (the common iliac arteries).

apnoea Absence of breathing.

ARDS Acute (or adult) respiratory distress syndrome. A sudden onset of respiratory fail-

1. These are terms used in the manual and during presentations and skill stations in the course. The definitions are couched in simple language to give the non-medical participant a better understanding of the course.

2. Taken and adapted from Emergency Care: a textbook for paramedics, Eds Ian Greaves, Tim Hodgetts and Keith Porter, with approval from WB Saunders 1997. 
ure often associated with the critically ill casualty and may frequently require ventilatory support.

artery Blood vessel supplying tissues with oxygenated blood.

assisted ventilation The boosting of tidal volume in a spontaneously breathing casualty.

atelectasis Failure of part of the lung to expand.

auscultation Listening and interpreting sounds as heard through the stethescope.

axilla The armpit.

Battle's sign Bruising behind the ear in the mastoid region due to a basal skull fracture.

Beck's triad The three principal physical signs of cardiac tamponade: muffled heart sounds, hypotension and neck vein distension.

bifurcate To split in two.

blood pressure The pressure exerted by the circulating blood on the arteries as it is pumped around the circulation.

bradycardia An unusually slow pulse rate, generally understood to be less than 60 beats/min, though this may be normal in a fit, young individual.

bronchus The division of the trachea leading down to the alveoli; the first two divisions after the trachea are known as the right and left main bronchi.

burn An injury caused by extremes of temperature - hot or cold. Classified as fullthickness or partial-thickness, depending how deep is the extent of tissue damage. Other causes of burns include chemicals and electricity.

carbon dioxide retention An increase in the concentration of carbon dioxide within the blood stream, often secondary to respiratory failure.

carbon monoxide A colourless, odourless gas. It has the ability to combine with haemoglobin in an almost irreversible manner, displacing oxygen and causing hypoxia of the tissues.

cardiac output The volume of blood pumped out by the heart in 1 minute. It is derived from the stroke volume (the amount of blood pumped per heartbeat) multiplied by heart rate.

cardiac tamponade A collection of fluid within the pericardial sac that leads to a reduction in the output of the heart.

cartoid arteries The two major arteries that can be felt in the neck giving arterial supply to the face and brain.

catheter Any flexible tube that may be placed within a body cavity to drain or instil fluids, (for example, urinary catheter for draining the bladder).

cephalo- Relating to the head.

cerebral oedema An increase in the fluid around the cells in brain tissue with resultant swelling. A number of causes including head injury (the most common). It may present with signs of raised intracranial pressure owing to the closed nature of the skull.

cerebral perfusion pressure A measure of the circulatory pressure to the brain. It can be calculated as the mean arterial pressure minus the intracranial pressure.

cerebro- Relating to the brain.

chin-lift A manoeuvre to open the airway by pulling forward the point of the chin.

clavicle (Collar bone) The supporting bone articulating between the scapula and the sternum.

coagulopathy An abnormal condition characterised by a decreased ability of the blood to clot. In trauma, this may be caused by continual bleeding and an inadequate replacement of coagulation factors after large blood loss.

colloid A solution used to increase circulating volume by intravenous transfusion, which contains proteins and large molecules, (for example, hetastarches, dextrans or albumin). coma Deep unconsciousness with no response to vocal or painful stimuli and absence of spontaneous eye movement.

concussion A limited period of unconsciousness caused by injury to the head.

conjunctiva The membrane that lines the eyelids and the sclera of the eye.

contralateral On the opposite side.

contrecoup The head injury sustained on the opposite side of the head to the site of the blow. It results from the movement of the soft brain against the cranium on the opposite side.

contusion The result of a blow to the body which fails to break the skin but produces swelling, bruising and pain.

cornea The transparent covering over the anterior part of the eye.

cranio- Relating to the skull.

cranium The bony framework of the skull. cricoid The last complete ring of cartilage in the upper airway before the horseshoe-like cartilages of the trachea.

Cricothyroidotomy Puncturing of the cricothyroid membrance in order to create an emergency airway. This may be done using a thick needle, or a formal surgical incision may be made.

crystalloid Solution used for intravenous fluid replacement containing only electrolytes and water.

CSF Cerebrospinal fluid: the fluid surrounding the brain and spinal cord.

cubital Relating to the elbow (for example, antecubital fossa).

cutaneous Pertaining to the skin.

cyanosis The blue colour seen at the periphery or centrally caused by the inadequate oxygenation of blood, which thus contains too much deoxyhaemoglobin which has a bluish colour.

dead space The area of the lung that is in contact with oxygen but is not being perfused with blood; also applied to the volume of air that is contained within the trachea and the bronchi which never reaches the gas 
exchange surface.

diaphragm The musculotendinous sheet that separates the thoracic cavity from the abdominal cavity.

diastole The period of time during the cardiac cycle when the heart is relaxed and blood enters the atria or ventricles.

diastolic blood pressure The systemic blood pressure during diastole. It rises slightly in the early phases of hypovolaemic shock. digit A finger or toe.

dislocation The displacement of any joint from its normal anatomical site and relationship with the surrounding tissues and bones. distal Further away along the body or a limb from a reference point.

dorso- Relating to the back or posterior aspect of the body or limb.

dorsum A structure on the back of a body or limb (for example, the dorsum of the hand). dura mater The thickest and outermost of the three layers of membrance that surround the brain and spinal cord. In the head this layer is fused to the cranial vault and is indistinguishable from the periosteum. Bleeding between the dura and the bone following head injury may lead to an extradural haematoma which may have potentially fatal results.

dyspnoea Shortness of breath whether as a result of disease or as a result of excessive exercise.

ecto- Prefix meaning on the outside.

electrocardiogram (ECG) A trace of the electrical activity of the heart normally taken as 12 differing views of the heart. Used to diagnose abnormalities of the heart such as arrhythmias and myocardial infarction.

endotracheal tube A large-bore tube used to secure an airway or to administer positive pressure ventilation.

enophthalmos A condition in which the eye is abnormally sunken into the socket for example following fracture of the floor of the orbit.

epidermis The superficial layers of the skin: the outer cornified layer and a deeper living layer.

epiglottis Protective structure that overhangs the larynx and hinges backwards during swallowing to prevent food entering the larynx.

exo- Prefix meaning outside.

extension Movement between two bones, generally in a direction to increase the angle between them.

extradural Literally, outside the dura; particularly refers to the cerebral bleed associated with a temporal fracture where the middle meningeal artery bleeds into the extradural space compressing the cerebral substance.

fascia (Latin, a band or sash) Fibrous connective tissue found in a variety of sites in the body, often wrapped around structures such as muscle or as a lining below fat and skin. femur The thigh bone.

fibula The small outer (lateral) bone of the lower leg.

flail chest A chest wall that as a result of trauma has two rows of multiple rib fractures leading to paradoxical movement.

flexion Movement between two bones, generally in a direction to decrease the angle between them.

fracture An injury to the bone in which the continuity of the bone is broken. There are a variety of classifications, but one important feature of note is whether the skin has been broken over the fracture site (compound or open fracture) or not (closed).

gag reflex The reflex occurring in response to irritation of the pharynx.

gangrene Death of tissue, often due to inadequate blood supply as the main cause, though a variety of other conditions such as cold injury may also cause gangrenous changes. Often divided into wet or dry gangrene: typically, wet gangrene follows major tissue damage such as crush injury.

Glasgow Coma Scale A swift method for assessing and monitoring the conscious level of a patient. It is based on the eye response, the verbal response and motor response.

glosso- Relating to the tongue.

greenstick fracture An incomplete fracture, usually in children, where the bone cortex is bent and deformed rather than disrupted.

guarding The contraction of the abdominal muscles to protect a painful area from the pressure of an examining hand.

haematoma A collection of blood within the tissues; this may be as a direct result of trauma or of surgery.

haematuria Passage of blood in the urine. haemoglobin The red pigment found in red blood cells that is responsible for the carriage of oxygen around the body.

haemorrhage The loss of blood - a term normally referring to rapid loss of a large quantity of blood either internally or externally.

haemothorax The filling of a pleural cavity with blood, usually after trauma to the chest or following thoracic surgery.

hemi- Prefix meaning half.

hepatic, hepato- Pertaining to the liver. hyper- Prefix meaning increased.

hypercapnia (hypercarbia) A high level of carbon dioxide in the blood.

hypertension Disorder of blood pressure control that results in an elevated blood pressure.

hypervolaemia A larger than required circulating volume, generally the result of overtransfusion, though overdrinking (particularly in the presence of reduced renal function) may also give rise to it.

hypo- Prefix meaning low or inadequate.

hypotension A low blood pressure which is inadequate for perfusion of the vital organs. This may be as a result of abnormalities of 
the heart, side effects of drugs in excess or loss of circulating fluid.

hypothermia A pathologically low body temperature (normally defined as below $35^{\circ} \mathrm{C}$ ), most frequently due to exposure to cold conditions.

hypovolaemia A state of low blood volume. See also Hypovolaemic shock.

hypovolaemic shock The state of inadequate perfusion (and therefore oxygenation of the tissues) due to a fall in the circulating volume (usually due to blood loss) and a subsequent fall in blood pressure. Excessive loss of body fluids due to other causes (for example, diarrhoea) can also occasionally cause this.

hypoxaemia Low level of oxygen in the blood.

hypoxia A fall in the concentration of oxygen in cells. This may result from inadequate oxygenation due to a decrease in atmospheric oxygen, inadequate respiration (due to a reduction in the respiratory drive, an obstruction of the respiratory tract or lung injury) or inadequate circulation (for example, shock).

ileus Cessation of gut mobility with resulting distension.

infra- Prefix meaning below or beneath.

infusion The introduction of a drug or fluid directly into the venous system.

inter- Prefix meaning between.

intermittent positive pressure ventilation Ventilation of a casualty who is not breathing, using a positive pressure system. intravenous Into a vein (administration of fluid or drugs).

intubation The passage of an endotracheal tube through the mouth or nose to secure an airway to the trachea or allow the passage of an anaesthetic agent.

ipsi- Prefix meaning the same (for example, ipsilateral, on the same side).

IRT Incident Response Team.

ischaemia An inadequate flow of blood to part of the body, caused by constriction or blockage of the blood vessels supplying it.

isotonic In medical terms, having the same osmotic pressure as extracellular fluid.

jaw-thrust A manoeuvre to open the airway by pushing the mandible forward, normally with the fingers behind the angle of the mandible.

joint A junction between two bones. This may be classified in a number of ways, for example by its structure (for example, a ball and socket joint such as the hip) or by its type (for example, fibrous, cartilaginous or synovial).

jugular Pertaining to the neck (for example, jugular vein, the major vein running on the right and left of the neck alongside the cartoid artery draining blood from the face and head.

lactic acid One of the waste products of protein and carbohydrate metabolism. It may accumulate in certain conditions to cause a metabolic acidosis.

laparotomy Surgical exploration of the abdominal cavity.

laryngo- Relating to the larynx.

laryngoscope An instrument for examining the larynx or for aid in viewing the larynx to assist the passage of an endotracheal tube.

larynx The upper part of the windpipe (voicebox)

lateral A position on the body further from the midline relative to another point.

ligate To tie off (with a ligature).

log roll Manoeuvre to move a casualty to expose the back but maintaining in-line stability of the whole spine.

longitudinal Along the long axis of the body.

lumbar (Latin, lumbus, the loin) General term referring to the lower back between the thorax and the pelvis, (for example, lumbar spine).

malleolus (Latin, a little hammer) Rounded, bony protuberances found on the inner (medial) and outer (lateral) aspect of the ankles.

mandible The lower bone of the jaw.

medial Towards the midline.

mediastinum (Latin, a middle partition) The middle cavity of the thorax within which the heart, the trachea (and its bifurcation), the great vessels and the oesophagus are contained.

meninges The membranes surrounding the brain substance, which become inflamed in meningitis. They comprise the dura mater, pia mater and arachnoid mater.

midclavicular line The imaginary line that runs vertically from the midpoint of the clavicle.

miotic A drug that causes the pupil to constrict, for example, pilocarpine.

mydriatic A drug that causes the pupil to dilate, for example, atropine.

myo- Relating to muscle.

narcotic $A$ drug that has pain-relieving properties and causes sleep.

nasal airway (nasopharyngeal airway) $\mathrm{A}$ flexible piece of tubing designed to pass through the nose into the pharynx to maintain an airway.

necrosis The death of tissue, often due to ischaemia.

neurogenic shock The condition of low blood pressure due to an injury to the spinal cord resulting in widespread dilation of blood vessels.

oedema An abnormal collection of fluid around tissue and cells or within tissue spaces.

oesophagus The muscular structure that carries food from the oropharynx to the stomach, linking the two structures.

open pneumothorax The collapse of a lung with entry of air into the pleural space and direct communication of the pleural space with the external environment. Also known as a 'sucking' chest wound. 
ophthalmo- Relating to the eye.

opiates Morphine and related drugs which may be used for pain relief. They have a tendency to cause respiratory despression and nausea. They may be administered subcutaneously, intramuscularly or intravenously.

oral airway A plastic or rubber device such as the Guedel airway designed to maintain an open airway in the unconscious or anaesthetised casualty. Also known as an oropharyngeal airway.

osteo- Relating to bone or bony.

oximeter A device that measures the saturation of the blood stream with oxygen by attaching a light transmitting and receiving probe to the skin.

oxygen A colourless and odourless gas which is vital for cellular metabolism. In conditions of shock and respiratory deficiency its supplementation forms an important part of therapy.

oxygen mask A device which is strapped to the face and used for administering supplementary oxygen.

paediatrics That branch of medicine specialising in the study and care of sick children. palate The dividing tissue forming the roof of the mouth separating the oral and nasal cavities. It is split into the hard (bony) palate anteriorly and soft palate posteriorly.

palpation To examine a casualty by feeling with the hand(s).

pancreas A large glandular retroperitoneal structure lying across the posterior midline of the abdomen.

paradoxical breathing Generally associated with traumatic damage to the chest, resulting in instability of the chest wall such that on inspiration the chest wall collapses in and the lung fails to expand.

paraesthesia The sensation of 'pins and needles' or tingling in the extremities.

pectoral (Latin pectus, the breast) Relating to the breast - usually the muscles (for example, pectoralis major).

perfusion The passage of blood through a tissue at a rate adequate to supply it with necessary nutrients and remove toxic metabolites.

peri- Prefix meaning around.

pericardial tamponade A collection of blood within the pericardial sac which prevents effective contraction of the heart.

pericardiocentesis The aspiration of fluid or blood from a pericardial effusion.

pericardium The fibrous sac that surrounds the heart. Fluid collecting within this sac but outside the heart as a result of either inflammation of the sac or trauma may lead to pressure on the heart and reduction in cardiac output.

perineum The area between the anal opening and the urethra perforated by the vaginal opening in the female.

peritoneum The lining membrane of the abdominal cavity, enfolding all the abdom- inal cavity. Organs in the abdomen may be within the peritoneal cavity or retroperitoneal (behind the peritoneal cavity): examples of the latter are the kidneys and pancreas. peritonitis Inflammation of the abdominal cavity due to bacterial, chemical or other causes. The most common causes include appendicitis, perforation or an ulcer or perforation of the small or large bowel.

pH The measure of acid and base balance within the body. It is a measure of the concentration of hydrogen ions present in the blood (the $\log$ of the reciprocal of the hydrogen ion concentration). Normal body $\mathrm{pH}$ is 7.4. In cases of acidosis (metabolic or respiratory) the $\mathrm{pH}$ falls. In alkalotic conditions (metabolic or respiratory alkalosis) the $\mathrm{pH}$ rises.

pharynx The throat. It is divided into the nasal cavity (nasopharynx), the oral cavity (oropharynx) and the larynx (laryngopharynx).

phrenic nerve The nerve supplying the diaphragm (motor and sensory).

plasma expander A substance given intravenously that is used to expand plasma volume or increase the oncotic (osmotic) pressure.

pleura The lining tissue that coats the inside of the thoracic cavity, the mediastinal contents and the lungs, allowing movement of the lungs relative to the thoracic wall.

pneumothorax A collection of air within the pleural space leading to collapse of the lung. This may be caused by a spontaneous leak from the lung or from perforation of the thoracic wall (for example, in stabbing). When a leak exists with a 'one-way valve' effect, the pressure of the pneumothorax may increase, leading to distortion of the mediastinum, decreased venous return and collapse: this is known as a tension pneumothorax.

popliteal fossa The fossa found at the back of the knee through which in its depth the major vascular and nervous tissues run to the calf and foot.

postero- Prefix meaning back.

pre- Prefix meaning in front of.

pre-load The pressure that causes ventricular filling of the heart.

priapism Persistent penile erection usually secondary to spinal cord injury.

pro- Prefix meaning before.

proptosis Forward displacement of the eye. proximal Usually referring to a point on a limb which is closer to the trunk than another point, (for example, the elbow is more proximal than the fingers).

pubis One of the three paired bones (including the ischium and ilium) that constitute the pelvis.

pulmonary Pertaining to the lungs and respiratory system.

pulmonary oedema The accumulation of excess fluid, firstly within the interstitial tissues of the lung and in severe cases within 
the actual alveoli of the lungs. Most commonly caused by congestive cardiac failure, though also associated with other acute conditions such as following acute head injury, near drowning and following inhalation injuries.

pulse The expansion and contraction of an artery as a result of the intermittent flow of arterial blood; can be detected by palpatation or other means.

pulse pressure The difference between the systolic and diastolic blood pressure. A reduction in the pulse pressure as a result of an increase in the distolic pressure may be one of the first and subtle signs of shock.

quadri- Prefix meaning four.

radial pulse The pulse of the radial artery which can be felt in the wrist; because of the ease with which it can be felt, it is the most frequently palpated.

radiograph An X-ray image.

rebound tenderness The pain felt on sudden release of a hand palpating the abdomen. It is seen in conditions of inflammation of the peritoneum.

rectum The terminal portion of the large bowel where faeces is stored prior to defacation.

referred pain Pain felt at one site but caused by disease at another anatomical site, (for example, hip disease may present with knee pain).

reservoir bag A component of anaesthetic apparatus that is used as a store for ventilatory gases between ventilatory cycles.

respiration 1 . The process of inhaling oxygen-rich atmospheric air and exhaling carbon dioxide-enriched air. 2. At a cellular level, the process of cellular exchange of oxygen as a fuel, metabolising it into carbon dioxide and excreting it.

respiratory acidosis The condition of increased carbon dioxide and carbonic acid (thus an increased hydrogen ion concentration) due to failure of excretion of carbon dioxide by the lungs. This may be due to suppression of the respiratory centre in the brain (for example, head injury, drugs, and so on) or lung disease.

respiratory alkalosis A condition characterised by a decrease in the concentration of carbon dioxide in the blood stream. Like respiratory acidosis it may be caused by lung disease through hyperventilation and excess excretion of carbon dioxide (for example, asthma or pneumonia). Other causes may include drugs and a variety of medical conditions.

retina The light-absorbing surface at the back of the eye.

retro- Prefix meaning behind.

retroperitoneum The potential space lying behind the peritoneum in which the retroperitoneal organs may be found (for example, the pancreas and kidneys).

rule of nines An approximate formula that may be used to calculate the surface area of a burn: $9 \%$ is allocated to each arm and the head; $18 \%(2 \times 9)$ to each leg and the front and back of the trunk; and $1 \%$ to the perineum.

sacrum The penultimate segment of the lower bony spine. It lies between the two hipbones, articulating with them via the sacroiliac joints.

sagittal Anatomical term describing the plane that runs from the front to the back of the body.

scapula The bone forming the shoulder blade.

semi- Prefix meaning half.

septic shock A form of shock secondary to the release of toxins from certain bacteria when they infect a patient. These cause decrease in the vascular resistance and a drop in blood pressure. Fever, an increased respiratory rate and confusion may also be features.

serum The thin clear fluid that is left behind after blood has clotted or after plasma has been allowed to form a clot.

shock Reduced perfusion of tissues inadequate to maintain their metabolic rate and oxygenation. It may have many causes, which fall into five main groups: hypovolaemic, septic, cardiogenic, spinal and anaphylactic.

skin traction A method for attaching a bandage or material (either adhesive or nonadhesive) to the skin of a limb in order to apply a corrective force reducing a fracture or orthopaedic deformity.

sphygmomanometer A device for measuring the arterial blood pressure.

spinal cord injury Traumatic damage of the spinal cord. The results depend on the level of the cord where the injury occurs and the severity of the cord damage. Cord damage below $\mathrm{C} 5$ and above $\mathrm{T} 1$ is associated with quadriplegia, while damage below $\mathrm{T} 1$ produces paraplegia. The effects depend on the severity of the damage and may range from temporary to permanent. Transection of the cord is associated with spinal shock characterised by warm peripheries, low blood pressure and absence of sensation and movement below the level of the injury.

spine The collective term for the group of bones which articulate together to form the backbone.

splanchnic (Greek splanchnos, an entrail) Relating to the viscera.

spleen A vascular organ in the upper left quadrant of the abdomen. Its role is primarily producing cells for the lymphoreticular system. Traumatic damage may result in severe haemorrhage owing to its vascular nature.

splint Any device that may be used to immobilise an injured part of the body.

sternum The breastbone. sub- Prefix meaning under.

subarachnoid haemorrhage The presence of blood within the subarachnoid space. 
subcutaneous emphysema (See surgical emphysema).

supination The movement of a limb or body towards the supine position, (that is, flat on the back with hands in a palms upward position).

supra- Prefix meaning above.

surgical emphysema The presence of gas in the subcutaneous or deep tissues of the body that has been forced there due to a leak of air from the lungs (rarely the gut). It may indicate the presence of a penetrating neck wound or pneumothorax, for example.

suture A material used to join surgical wounds or damaged tissues.

systole The part of the cardiac cycle in which the heart contracts and blood is expelled into the aorta.

tachycardia An abnormally fast pulse rate, generally defined as a pulse rate above 100 beats per minute.

tamponade Abnormal pressure on part of the body.

tension pneumothorax see Pneumothorax.

thoraco- Relating to the chest.

thoracostomy The formation of a hole in the thoracic wall to enable the passage of a chest drain. In the emergency setting this may be performed by the insertion of a needle (needle thoracocentesis).

thoracotomy The surgical incision and opening of the thoracic cavity.

thorax, thoracic cage The upper body cavity containing the principal organs of respiration and circulation. Bounded by the sternum at the front, the thoracic vertebrae at the back and the ribs around the sides.

thready pulse A pulse that is weak or difficult to feel: may be related to a small pulse volume (pulse pressure) secondary to shock.

tibia The main weightbearing bone of the lower limb below the knee joint.

trachea The main air passage connecting the lungs the oropharnyx and nasopharynx.

tracheostomy The surgical creation of an artificial airway by incising the trachea. This may be performed to aid long-term ventilation on an intensive care unit or as an emergency to circumvent an airway problem.

traction The placement of a limb or body part under tension; often used in orthopaedics to correct deformity, realign broken bones and as temporary method of pain relief at a fracture site.

trans- Prefix meaning across or over.

transfusion The replacement of lost blood with stored blood. This is most commonly from an unrelated donor and as such, the blood requires cross-matching prior to transfusion.

transfusion reaction The response of the body to a transfusion of unmatched and incompatible blood. Fever, bronchoconstriction and renal failure may result. At its worst profound shock may develop. transverse At right angles to the long axis of the body (this plane is also at right angles to the coronal and sagittal planes).

triage The classification and sorting of casualties into groups based on the severity of injury and chance of survival. Successful triage aims to do the most for the most, even if resources are limited.

ulna The long bone of the forearm that runs on the inner aspect from the elbow to the wrist. At the elbow it forms the olecranon.

ultrasound High-frequency sound waves $(>20,000 \mathrm{~Hz})$.

unconsciousness The inability to sense and respond to external stimuli, due to a variety of causes.

uni- Prefix meaning one.

universal donor The blood group $\mathrm{O} R \mathrm{Rh}$ negative which can be given to almost any individual in the event of an emergency with minimal risk of a transfusion reaction.

urethra The terminal passage from the bladder for voiding of urine. In females it is a short tube that lies anterior to the vagina; in males it is about $20 \mathrm{~cm}$ long and passes through the middle of the prostate gland before running the length of the penis to the external meatus.

Valsalva manoeuvre Breathing out against a closed glottis to increase intrathoracic pressure.

vasoconstriction The narrowing of the blood vessels, particularly the aterioles and veins. This may be accomplished by a variety of stimuli and is useful in the response to shock or to control blood pressure.

vasodilatation The widening of the various small vessels (see vasoconstriction) brought about by relaxation of the smooth muscle in the walls of the vessels.

ventilation The process of exchanging gases from within the lung to the atmosphere.

ventilator $A$ device that may be used either to provide ventilatory support or to perform complete ventilation.

vertebra One of the constituent bones of the spinal column. It may be a cervical, thoracic, lumbar, sacral or coccygeal vertebra.

visceral Relating to any internal organ in a body cavity. Though often referring to organs of the abdominal cavity it can also refer to those of the thoracic cavity.

volume expander Any form of intravenous fluid that stays within the intravascular space, expanding it (normally refers to colloid, though blood is also classified as a volume expander).

whiplash injury Forced flexion/extension injury of the neck often seen following a road traffic accident.

xiphoid The lower part of the sternal bone, generally cartilagenous in nature. It may be used as an anatomical reference point to decide where to place the hands for CPR. zygoma An extension of the temporal bone of the head which forms the prominence of the cheek. 